\title{
A Necessary Characteristic Equation of Diffusion Processes Having Gaussian Marginals
}

\author{
Syeda Rabab Mudakkar \\ Centre for Mathematical and Statistical sciences, Lahore School of Economics, Lahore 53200, Pakistan \\ Correspondence should be addressed to Syeda Rabab Mudakkar, drrabab@lahoreschool.edu.pk
}

Received 2 January 2012; Accepted 6 February 2012

Academic Editor: Márcia Federson

Copyright (C) 2012 Syeda Rabab Mudakkar. This is an open access article distributed under the Creative Commons Attribution License, which permits unrestricted use, distribution, and reproduction in any medium, provided the original work is properly cited.

The aim of this work is to characterize one-dimensional homogeneous diffusion process, under the assumption that marginal density of the process is Gaussian. The method considers the forward Kolmogorov equation and Fourier transform operator approach. The result establishes the necessary characteristic equation between drift and diffusion coefficients for homogeneous and nonhomogeneous diffusion processes. The equation for homogeneous diffusion process leads to characterize the possible diffusion processes that can exist. Two well-known examples using the necessary characteristic equation are also given.

\section{Introduction and Preliminaries}

The characterization of drift and diffusion coefficients by considering the marginal density of stochastic process is one of the known methods of estimating the actual underlying process and to construct new diffusion processes [1-6]. In this paper, we will consider the forward Kolmogorov equation and apply the Fourier transform operator to prove necessary characteristic equation between drift and diffusion coefficients for a homogeneous one-dimensional diffusion process when the marginal density is Gaussian. This equation characterizes the possible diffusion processes. A similar equation is proved for a nonhomogeneous diffusion process. However, the idea is motivated by Hamza and Klebaner [6], who constructed an entire family of non-Gaussian martingales given that the marginals are Gaussian.

Before stating our main result, we introduce some of the common notations, assumptions, and key facts used in this work.

(i) $a(z)$ and $a(z, t)$ denote the drift coefficient of homogeneous and nonhomogeneous one-dimensional Itô-diffusion process $X=\left\{X_{t}, t \geq 0\right\}$ given $X_{t}=z$. Similarly, $\sigma(z)$ 
and $\sigma(z, t)$ denote the diffusion coefficient of homogeneous and non-homogeneous one-dimensional Itô-diffusion process $X=\left\{X_{t}, t \geq 0\right\}$ given $X_{t}=z$.

(ii) $p_{t, t_{0}}(z \mid y)$ denotes the density function of $\left(X_{t}=z \mid X_{t_{0}}=y\right)$ with mean $\mu_{t}$ and variance $\gamma_{t}$.

(iii) $C_{0}(\mathbb{R})$ denotes the set of all continuous functions with domain $\mathbb{R}, C_{0}^{2}(\mathbb{R})$ denotes the set of all continuous twice-differentiable functions with domain $\mathbb{R}$, and $C_{0}^{2,1}(\mathbb{R})$ denotes the set of all continuous twice-differentiable functions in the first variable and continuous differentiable functions in the second variable with domain $\mathbb{R}^{2}$.

(iv) $f_{x}^{\prime}$ or $f^{\prime}(x)$ denotes the first derivative of function $f$ with respect to $x$.

(v) The conditional density $p_{t, t_{0}}(z \mid y)$ is bounded by $e^{-k z^{2}}$.

(vi) The assumption $t_{0}>0$ is considered due to the continuity problem at $t=0$. It means that if the conditional probability distribution of the increments is considered from time 0 , then the method we use is not able to identify those drift and diffusion coefficients that lead us to construct continuous diffusion process.

(vii) $f(z):=e^{i z x}$ is considered to use the uniqueness property of the characteristic function.

We need the following standard forward Kolmogorov equations introduced in [7].

Definition 1.1. Let the homogeneous diffusion process $X=\left\{X_{t}, t \geq 0\right\}$ satisfy the one-dimensional stochastic differential equation (SDE)

$$
d X_{t}=a\left(X_{t}\right) d t+\sigma\left(X_{t}\right) d B_{t}
$$

where $\left\{B_{t}, t \geq 0\right\}$ is a standard Brownian motion. Let $f(x)$ be bounded twice continuously differentiable in $x$ (i.e., $C_{0}^{2}(\mathbb{R})$ ) function, then

$$
\frac{d}{d t}\left[E\left[f\left(X_{t}\right) \mid X_{t_{0}}=y\right]\right]=E\left[f^{\prime}\left(X_{t}\right) a\left(X_{t}\right)\right]+E\left[f^{\prime \prime}\left(X_{t}\right) \frac{\sigma^{2}\left(X_{t}\right)}{2}\right]
$$

where $y$ is any fixed point.

Definition 1.2. Let the non-homogeneous diffusion process $X=\left\{X_{t}, t \geq 0\right\}$ satisfy the onedimensional stochastic differential equation (SDE)

$$
d X_{t}=a\left(X_{t}, t\right) d t+\sigma\left(X_{t}, t\right) d B_{t}
$$

Let $f(x, t)$ be bounded twice continuously differentiable in $x$ and continuously differentiable in $t$ (i.e., $\left.C_{0}^{2,1}(\mathbb{R})\right)$ function, then

$$
\frac{\partial}{\partial t}\left[E\left[f\left(X_{t}, t\right) \mid X_{t_{0}}=y\right]\right]=E\left[\frac{\partial f\left(X_{t}, t\right)}{\partial X_{t}} a\left(X_{t}, t\right)\right]+E\left[\frac{\partial^{2} f\left(X_{t}, t\right)}{\partial X_{t}^{2}} \frac{\sigma^{2}\left(X_{t}, t\right)}{2}\right],
$$

where $y$ is any fixed point. 
The conditions that ensure the existence and uniqueness of any stochastic differential equation, following terminology of [8], are as follows.

Definition 1.3. Let the diffusion process $X=\left\{X_{t}, t \geq 0\right\}$ satisfy (1.3), then

(1) drift and diffusion coefficients are said to be locally Lipschitz in $x$ and uniformly in $t$ if for every $T$ and $N$, there is a constant $K$ depending only on $T$ and $N$ such that for all $|x|,|y| \leq N$ and all $0 \leq t \leq T$,

$$
|a(x, t)-a(y, t)|+|\sigma(x, t)-\sigma(y, t)|<K|x-y|
$$

(2) the coefficients satisfy the linear growth condition if

$$
|a(x, t)|+|\sigma(x, t)| \leq K(1+|x|) .
$$

Definition 1.3 holds in the same way for homogeneous diffusion process.

\section{Main Results}

In Section 2.1, we obtain the characteristic equation between drift and diffusion coefficients under the assumption that marginals are Gaussian. In Section 2.2, we identify possible diffusion processes using the characteristic equation.

\subsection{Characteristic Equation}

We first prove the characteristic equation for homogeneous diffusion process and then extend our results when the starting position $X_{t_{0}}$ is arbitrary. Finally we prove the characteristic equation in case of non-homogeneous diffusion.

Theorem 2.1. Let the homogeneous diffusion process $X=\left\{X_{t}, t \geq 0\right\}$ satisfy the stochastic differential equation (1.1), $a\left(X_{t}\right) \in C_{0}(\mathbb{R}), \sigma\left(X_{t}\right) \in C_{0}^{2}(\mathbb{R})$, and both $a\left(X_{t}\right)$ and $\sigma\left(X_{t}\right)$ satisfy the linear-growth and local Lipschitz conditions:

(i) assume $\left(X_{t}=z \mid X_{t_{0}}=y\right) \sim N\left(y+\mu_{t}, \gamma_{t}\right)$ where $t_{0}>0$ and $t>t_{0}, \mu_{t} \in C_{0}(\mathbb{R})$ and $\gamma_{t} \in C_{0}(\mathbb{R})$, then the necessary equation on drift and diffusion coefficients obtained by conditioning on $X_{t}=z$ is given by

$$
a(z)=\mu_{t}^{\prime}+\frac{\left(\sigma^{2}(z)\right)_{z}^{\prime}}{2}+\left(z-y-\mu_{t}\right)\left(\frac{\gamma_{t}^{\prime}-\sigma^{2}(z)}{2 \gamma_{t}}\right)
$$

(ii) assume $X_{t_{0}}$ is arbitrary and $E\left[p_{t}\left(z \mid X_{t_{0}}\right)\right] \sim N\left(\mu_{t}, \gamma_{t}\right)$, where $t_{0}>0$ and $t>t_{0}, \mu_{t} \in C_{0}(\mathbb{R})$ and $\gamma_{t} \in C_{0}(\mathbb{R})$, then the necessary equation on drift and diffusion coefficients by conditioning on $X_{t}=z$ is (2.1). 
Proof (i). Using (1.2),

$$
\begin{aligned}
\frac{d}{d t}\left(\int_{-\infty}^{\infty} f(z) p_{t, t_{0}}(z \mid y) d z\right)= & \int_{-\infty}^{\infty}\left(\frac{d f(z)}{d z} a(z)\right) p_{t, t_{0}}(z \mid y) d z \\
& +\int_{-\infty}^{\infty}\left(\frac{\left(d^{2} f(z) / d z^{2}\right) \sigma^{2}(z)}{2}\right) p_{t, t_{0}}(z \mid y) d z
\end{aligned}
$$

Under the assumption

$$
\xi:=\left(X_{t}=z \mid X_{t_{0}}=y\right) \sim N\left(y+\mu_{t}, \gamma_{t}\right),
$$

which implies

$$
p_{t}(\xi)=p_{t, t_{0}}(z \mid y):=\frac{1}{\sqrt{2 \pi \gamma_{t}}} e^{-\left(z-y-\mu_{t}\right)^{2} / 2 \gamma_{t}},
$$

consider a function $f: \mathbb{R} \rightarrow \mathbb{R}$, that is,

$$
f(z):=e^{i z x}
$$

Thus

$$
\int_{-\infty}^{\infty} e^{i z x} p_{t, t_{0}}(z \mid y) d z=e^{\left(i x\left(y+\mu_{t}\right)\right)-\left(\left(x^{2} \gamma_{t}\right) / 2\right)}
$$

Substituting in (2.2) and simplification yields

$$
\frac{x}{2 \sqrt{2 \pi \gamma_{t}}} \int_{-\infty}^{\infty}\left(\sigma^{2}(z)-\gamma_{t}^{\prime}\right) e^{i z x} e^{-\left(z-y-\mu_{t}\right)^{2} / 2 \gamma_{t}} d z=\frac{i}{\sqrt{2 \pi \gamma_{t}}} \int_{-\infty}^{\infty}\left(a(z)-\mu_{t}^{\prime}\right) e^{i z x} e^{-\left(z-y-\mu_{t}\right)^{2} / 2 \gamma_{t}} d z
$$

Consider left-hand side of (2.7) and define

$$
G(z):=\left(\sigma^{2}(z)-\gamma_{t}^{\prime}\right) e^{-\left(z-y-\mu_{t}\right)^{2} / 2 \gamma_{t}}
$$

Clearly $G(z)$ is dominated by $e^{-K z^{2} / 2}$. Thus, integration by parts and using property of characteristic function, we obtain

$$
e^{-\left(z-y-\mu_{t}\right)^{2} / 2 \gamma_{t}}\left(\sigma(z)(\sigma(z))_{z}^{\prime}-\frac{\left(z-y-\mu_{t}\right)}{2 \gamma_{t}}\left(\sigma^{2}(z)-\gamma_{t}^{\prime}\right)\right)=\left(a(z)-\mu_{t}^{\prime}\right) e^{-\left(z-y-\mu_{t}\right)^{2 / 2 \gamma_{t}}}
$$


and it further implies that

$$
a(z)=\mu_{t}^{\prime}+\frac{\left(\sigma^{2}(z)\right)_{z}^{\prime}}{2}-\frac{\left(z-y-\mu_{t}\right)}{2 \gamma_{t}}\left(\sigma^{2}(z)-\gamma_{t}^{\prime}\right)
$$

Proof (ii). Consider

$$
\begin{aligned}
E\left[E\left[f\left(X_{t+\Delta t}\right) \mid X_{t_{0}}\right]\right] & =E\left[\int_{-\infty}^{\infty} E\left(f\left(X_{t+\Delta t}\right) \mid X_{t}=z\right) p_{t}\left(z \mid X_{t_{0}}\right) d z\right] \\
& =\iint_{-\infty}^{\infty} E\left(f\left(X_{t+\Delta t}\right) \mid X_{t}=z\right) p_{t}\left(z \mid X_{t_{0}}=y\right) p_{t_{0}}(y) d z d y
\end{aligned}
$$

provided that $E\left[f\left(X_{t+\Delta t}\right)\right]<\infty$. By Fubini's theorem, interchanging the integrals gives

$$
\begin{aligned}
E\left[E\left[f\left(X_{t+\Delta t}\right) \mid X_{t_{0}}\right]\right] & =\int_{-\infty}^{\infty} E\left(f\left(X_{t+\Delta t}\right) \mid X_{t}=z\right) \int_{-\infty}^{\infty} p_{t}\left(z \mid X_{t_{0}}=y\right) \mathrm{p}_{\mathrm{t}_{0}}(y) d y d z \\
& =\int_{-\infty}^{\infty} E\left(f\left(X_{t+\Delta t}\right) \mid X_{t}=z\right) E\left(p_{t}\left(z \mid X_{t_{0}}\right)\right) d z .
\end{aligned}
$$

Now, using Taylor's expansion around $X_{t}=z$ and then Itô's formula, we reach the same forward Kolmogorov equation, that is,

$$
\frac{d}{d t}\left(E\left[E\left(f\left(X_{t}\right) \mid X_{t_{0}}\right)\right]\right)=\int_{-\infty}^{\infty}\left(f^{\prime}(z) a(z)+\frac{f^{\prime \prime}(z)}{2} \sigma^{2}(z)\right) E\left(p_{t}\left(z \mid X_{t_{0}}\right)\right) d z
$$

where

$$
\begin{aligned}
E\left[E\left(f\left(X_{t}\right) \mid X_{t_{0}}\right)\right] & =E\left(\int_{-\infty}^{\infty} f(z) p_{t}\left(z \mid X_{t_{0}}\right) d z\right) \\
& =\iint_{-\infty}^{\infty} f(z) p_{t}\left(z \mid X_{t_{0}}=y\right) p_{t_{0}}(y) d z d y \\
& =\int_{-\infty}^{\infty} f(z) \int_{-\infty}^{\infty} p_{t}\left(z \mid X_{t_{0}}=y\right) p_{t_{0}}(y) d y d z \\
& =\int_{-\infty}^{\infty} f(z) E\left(p_{t}\left(z \mid X_{t_{0}}\right)\right) d z
\end{aligned}
$$

and therefore,

$$
\frac{d}{d t}\left(\int_{-\infty}^{\infty} f(z) E\left(p_{t}\left(z \mid X_{t_{0}}\right)\right) d z\right)=\int_{-\infty}^{\infty}\left(f^{\prime}(z) a(z)+\frac{f^{\prime \prime}(z)}{2} \sigma^{2}(z)\right) E\left(p_{t}\left(z \mid X_{t_{0}}\right)\right) d z
$$


under the assumption

$$
E\left[p_{t}\left(z \mid X_{t_{0}}\right)\right]=\frac{1}{\sqrt{2 \pi \gamma_{t}}} e^{-\left(z-\mu_{t}\right)^{2} / 2 \gamma_{t}}
$$

The remaining proof follows the same arguments as (i).

Theorem 2.2. Let the non-homogeneous diffusion process $X=\left\{X_{t}, t \geq 0\right\}$ satisfy the stochastic differential equation (1.3), $a\left(X_{t}, t\right) \in C_{0}(\mathbb{R}), \sigma\left(X_{t}\right) \in C_{0}^{2,1}(\mathbb{R})$, and both $a\left(X_{t}, t\right)$ and $\sigma\left(X_{t}, t\right)$ satisfy the linear-growth and local Lipschitz conditions. Assuming $\left(X_{t}=z \mid X_{t_{0}}=y\right) \sim N\left(y+\mu_{t}, \gamma_{t}\right)$ where $t_{0}>0$ and $t>t_{0}, \mu_{t} \in C_{0}(\mathbb{R})$ and $\gamma_{t} \in C_{0}(\mathbb{R})$, then the necessary equation on drift and diffusion coefficients by conditioning on $X_{t}=z$ is given by

$$
a(z, t)=\mu_{t}^{\prime}+\frac{\left(\sigma^{2}(z, t)\right)_{z}^{\prime}}{2}+\left(z-y-\mu_{t}\right)\left(\frac{\gamma_{t}^{\prime}-\sigma^{2}(z, t)}{2 \gamma_{t}}\right)
$$

Proof. Consider (1.4) and treat it in an analogue manner as proof of Theorem 2.1 (i). We have omitted further details.

\subsection{Characterization of Possible Diffusion Processes}

We characterize the possible diffusion processes using (2.1). The following result explains possible solutions of (2.1).

Theorem 2.3. Suppose that the mean parameter of marginal density satisfies $\mu_{t}=0$, then under the assumption that marginals are Gaussian, (2.1) characterizes that the only possible solution exists when it is a diffusion coefficient, that is, $\sigma^{2}(z)=K$ or variance parameter of marginal density, that is, $\gamma_{t}=\gamma$ or $\sigma^{2}(z)=K$ and drift coefficient, that is, $a(z)=1 /(z-y)$.

Proof. Equation (2.1) can be written as

$$
\frac{\left(\sigma^{2}(z)\right)_{z}^{\prime}-2 a(z)}{2(z-y)}+\frac{\gamma_{t}^{\prime}}{2 \gamma_{t}}-\frac{\sigma^{2}(z)}{2 \gamma_{t}}=0
$$

Define

$$
G(z):=\frac{\left(\sigma^{2}(z)\right)_{z}^{\prime}-2 a(z)}{2(z-y)}, \quad \xi(t):=\frac{\gamma_{t}^{\prime}}{2 \gamma_{t}}, \quad K(t):=\frac{1}{2 \gamma_{t}}, \quad H(z):=\sigma^{2}(z),
$$

which yields

$$
G(z)+\xi(t)-K(t) H(z)=0
$$

Case 1. Differentiating (2.20) with respect to $t$ gives $\xi^{\prime}(t)-K^{\prime}(t) H(z)=0$. Clearly, $\xi^{\prime}(t)$ and $K^{\prime}(t)$ are not equal to zero. Hence, $\xi^{\prime}(t) / K^{\prime}(t)=H(z)$ which indicates that $H(z)=\sigma^{2}(z)=K$, where $K$ is any arbitrary constant. 
Case 2. Differentiating (2.20) with respect to $z$ gives $G^{\prime}(z)-K(t) H^{\prime}(z)=0$.

(i) If $H(z)=\sigma^{2}(z)=K$, then $H^{\prime}(z)=0$. Clearly $G^{\prime}(z)$ is equal to zero if $a(z)=1 /(z-y)$.

(ii) If $H^{\prime}(z)$ and $G^{\prime}(z)$ are not equal to zero, then $G^{\prime}(z) / H^{\prime}(z)=K(t)$. This implies that $K(t)=K$, which yields $\gamma_{t}=\gamma$, where $\gamma$ is any arbitrary constant which does not depend on $t$.

Example 2.4. Equation (2.1) characterizes the martingale case, that is, when drift coefficient $a(z)=0$, the process is given by

$$
X_{t}=X_{t_{0}}+\int_{t_{0}}^{t} K d B_{s}
$$

where $K$ is any arbitrary constant.

Proof. Assuming $a(z)=0$ in (2.7) gives

$$
\frac{x}{2 \sqrt{2 \pi \gamma_{t}}} \int_{-\infty}^{\infty}\left(\sigma^{2}(z)-\gamma_{t}^{\prime}\right) e^{i z x} e^{-\left(z-y-\mu_{t}\right)^{2} / 2 \gamma_{t}} d z=\mu_{t}^{\prime} \frac{-i}{\sqrt{2 \pi \gamma_{t}}} \int_{-\infty}^{\infty} e^{i z x} e^{-\left(z-y-\mu_{t}\right)^{2} / 2 \gamma_{t}} d z
$$

Clearly $x$ is arbitrary, which implies that

$$
\mu_{t}^{\prime}=0, \quad \text { or } \quad \mu_{t}=K
$$

Hence, from (2.1), we obtain

$$
\frac{\left(\sigma^{2}(z)\right)_{z}^{\prime}}{2(z-y-K)}+\frac{\gamma_{t}^{\prime}}{2 \gamma_{t}}-\frac{\sigma^{2}(z)}{2 \gamma_{t}}=0 .
$$

The remaining arguments follow from proof of Theorem 2.3 of Case 1.

Example 2.5. Suppose that variance parameter of the marginal density $\gamma_{t}$ has two values, mean of marginal density $\mu_{t}=0$, starting position of diffusion process $X_{t_{0}}=y$ and diffusion coefficient $\sigma^{2}(z)=K$, then (2.1) characterizes the Vasicek model given by

$$
X_{t}=y+\frac{K}{2} \int_{t_{0}}^{t}\left(X_{s}-y\right) d s+K \int_{t_{0}}^{t} d B_{s}
$$

Proof. Equation (2.1) can be written as

$$
\frac{2\left(a(z)-\sigma(z) \sigma^{\prime}(z)\right)}{(z-y)} \gamma_{t}=\gamma_{t}^{\prime}-\sigma^{2}(z)
$$


Define

$$
L(z):=\frac{2\left(a(z)-\sigma(z) \sigma^{\prime}(z)\right)}{(z-y)}
$$

and assume two different values of $\gamma_{t}$, that is, $\gamma_{t}=a_{t}$ and $\gamma_{t}=b_{t}$, which gives

$$
\begin{aligned}
& L(z) a_{t}+\sigma^{2}(z)=a_{t}^{\prime} \\
& L(z) b_{t}+\sigma^{2}(z)=b_{t}^{\prime} .
\end{aligned}
$$

Subtracting (2.28) from (2.29), we obtain

$$
L(z)=\frac{\left(b_{t}^{\prime}-a_{t}^{\prime}\right)}{\left(b_{t}-a_{t}\right)}=K \text { which does not depend on } z \text {. }
$$

Therefore,

$$
\sigma^{2}(z)=\gamma_{t}^{\prime}-K \gamma_{t}=C \text { thus }\left(\sigma^{2}(z)\right)_{z}^{\prime}=0
$$

By substituting values in (2.27), we obtain

$$
a(z)=\frac{K(z-y)}{2} \quad \text { when } \sigma^{2}(z)=C
$$

Hence,

$$
X_{t}=y+\frac{K}{2} \int_{t_{0}}^{t}\left(X_{s}-y\right) d s+K \int_{t_{0}}^{t} d B_{s}
$$

Remark 2.6. The variance of the marginal density, that is, $\gamma_{t}$, can have at most two different values.

\section{Conclusion}

We have proved the necessary characteristic equation for homogeneous and non-homogeneous one-dimensional diffusion processes, under the assumption that marginal density of the process is Gaussian. The possible diffusion processes that can exist are also characterized. Two well-known examples using our characteristic equation are given. However, we are still working on sufficient condition of the characteristic equation and about the construction of a new diffusion process whose diffusion coefficients do not satisfy usual Lipschitz and lineargrowth conditions, but still it exists. 


\section{Acknowledgments}

The author would like to thank the referee for his valuable comments and Dr. Sergey Utev for his invaluable guidance and support. Thanks are also due to Higher Education Commission of Pakistan and Lahore School of Economics, Pakistan for funding this research.

\section{References}

[1] H. G. Kellerer, "Markov-Komposition und eine Anwendung auf Martingale," Mathematische Annalen, vol. 198, pp. 99-122, 1972.

[2] Y. Aït-Sahalia, "Nonparametric pricing of interest rate derivative securities," Econometrica, vol. 64, no. 3, pp. 527-560, 1996.

[3] B. M. Bibby and M. Sørensen, "Simplified estimating functions for diffusion models with a highdimensional parameter," Scandinavian Journal of Statistics, vol. 28, no. 1, pp. 99-112, 2001.

[4] D. B. Madan and M. Yor, "Making Markov martingales meet marginals: with explicit constructions," Bernoulli, vol. 8, no. 4, pp. 509-536, 2002.

[5] B. M. Bibby, I. M. Skovgaard, and M. Sørensen, "Diffusion-type models with given marginal distribution and autocorrelation function," Bernoulli, vol. 11, no. 2, pp. 191-220, 2005.

[6] K. Hamza and F. C. Klebaner, "A family of non-Gaussian martingales with Gaussian marginals," Journal of Applied Mathematics and Stochastic Analysis, vol. 2007, Article ID 92723, 19 pages, 2007.

[7] A. Kolmogorov, "On analytic methods in the theory of probability," Uspekhi Matematicheskikh Nauk, no. 5, pp. 5-41, 1938.

[8] F. C. Klebaner, Introduction to Stochastic Calculus with Applications, Imperial College Press, London, UK, 2005. 


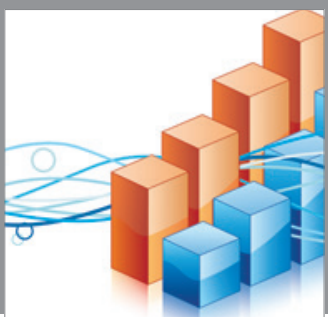

Advances in

Operations Research

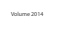

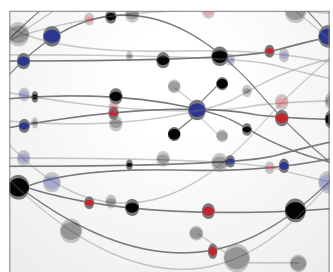

\section{The Scientific} World Journal
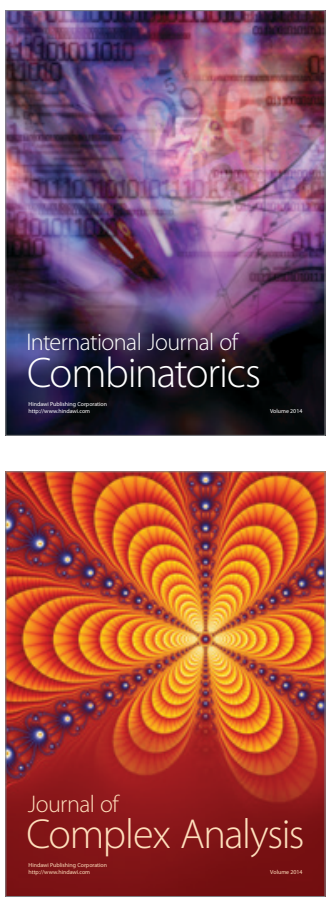

International Journal of

Mathematics and

Mathematical

Sciences
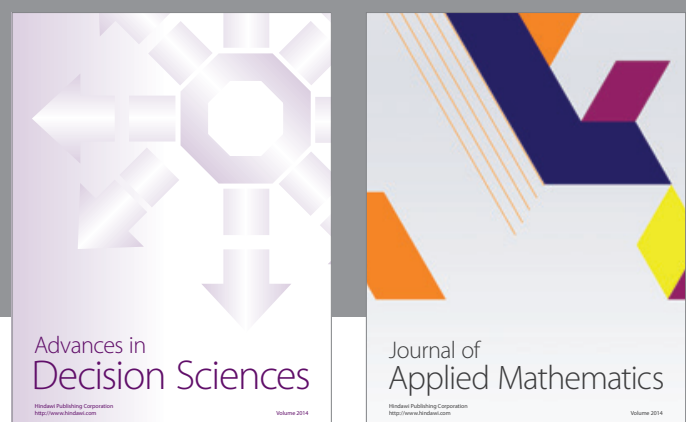

Journal of

Applied Mathematics
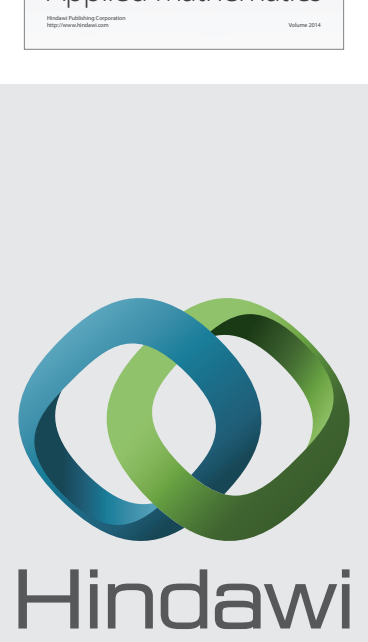

Submit your manuscripts at http://www.hindawi.com
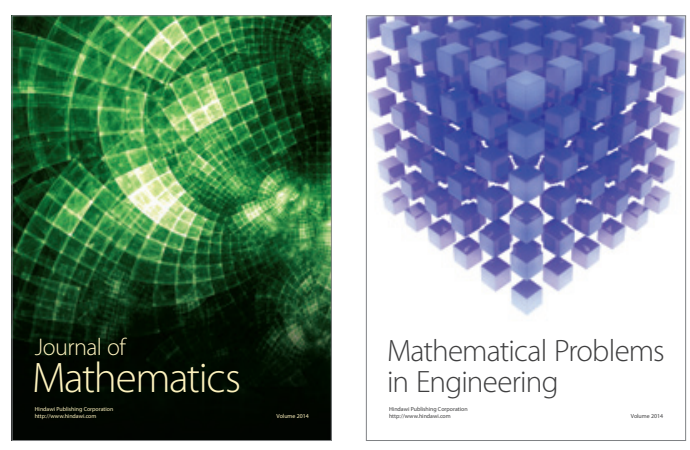

Mathematical Problems in Engineering
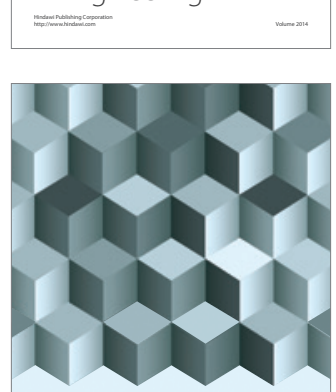

Journal of

Function Spaces
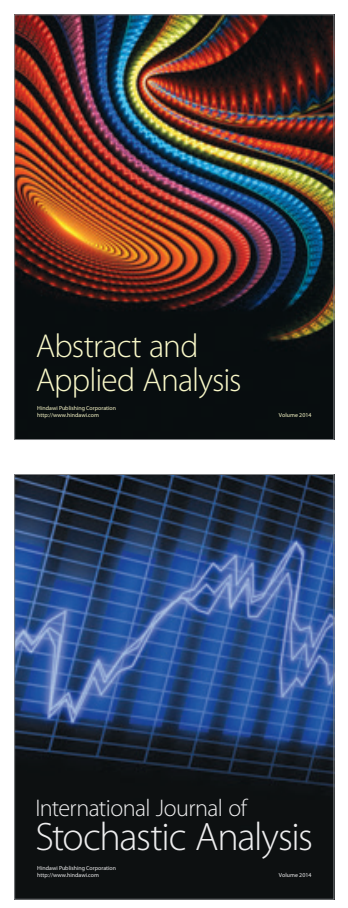

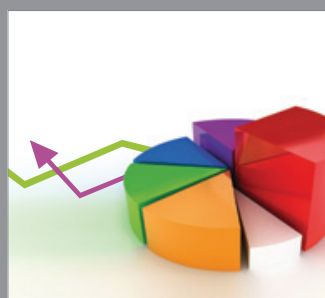

ournal of

Probability and Statistics

Promensencen
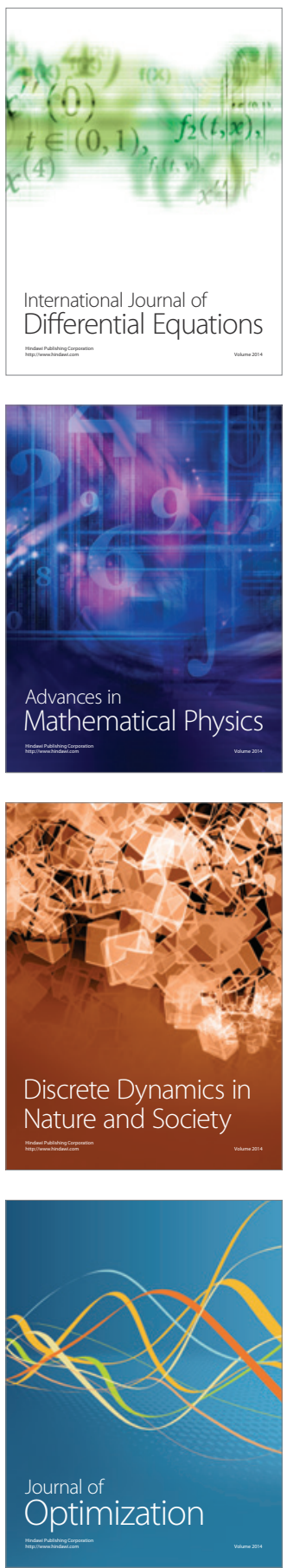